
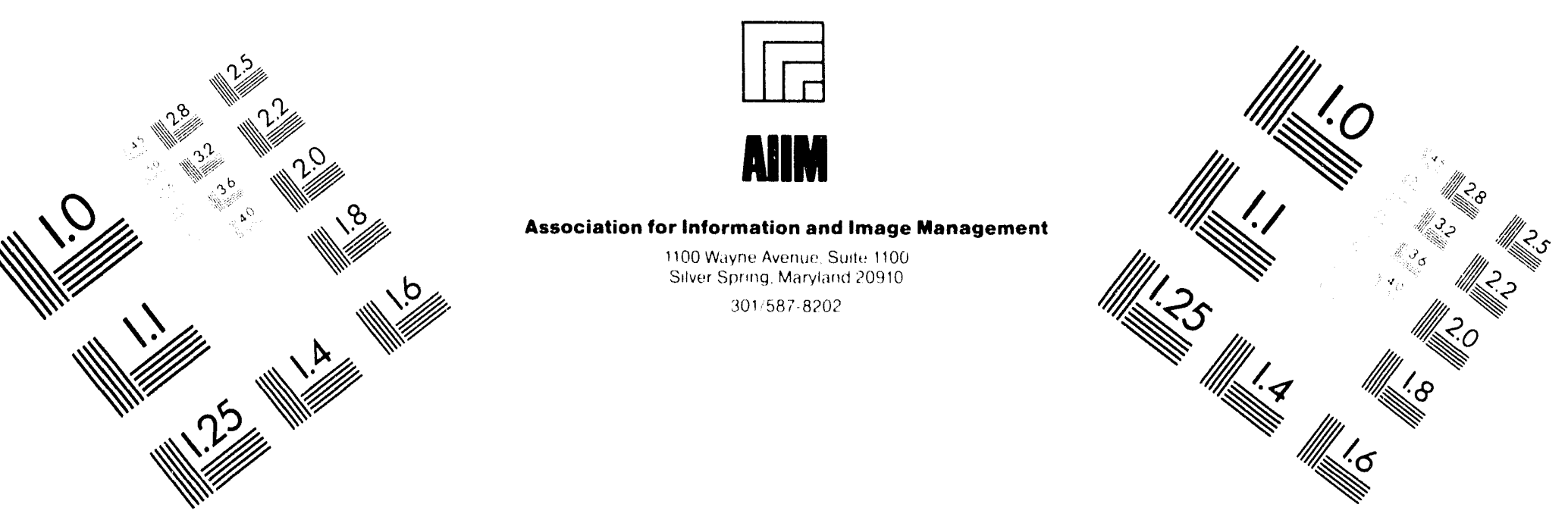

\title{
Centimeter
}

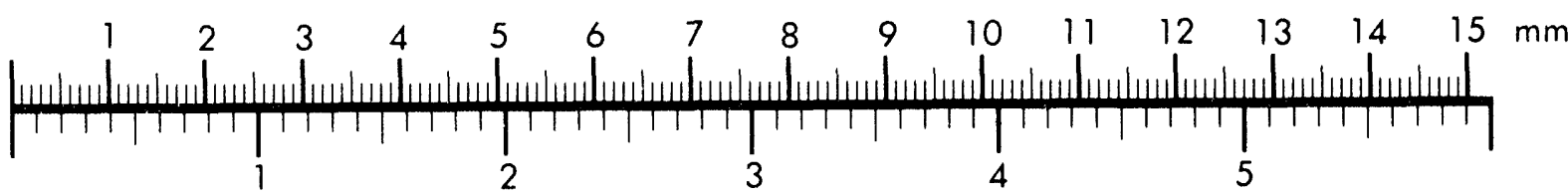

Inches
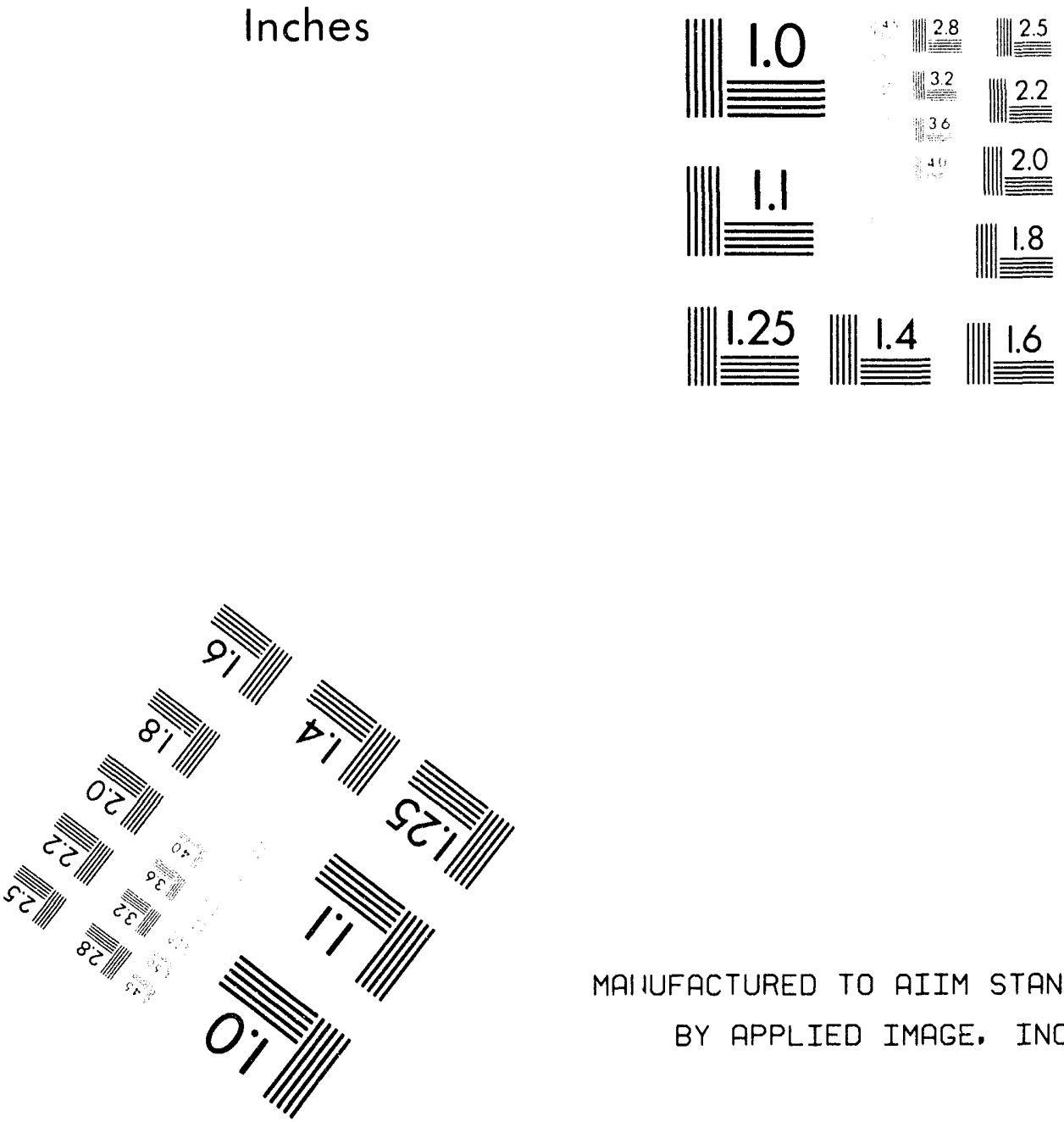

MAI IUFACTURED TO AIIM STANDARDS

BY APPLIED IMAGE. INC.

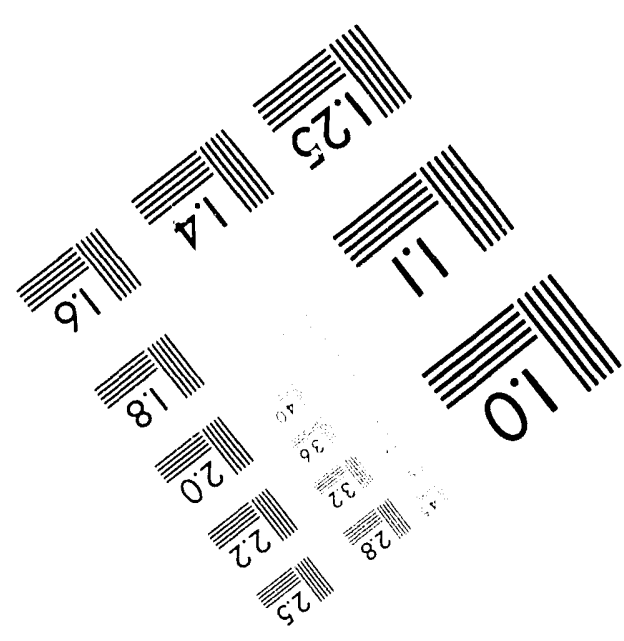



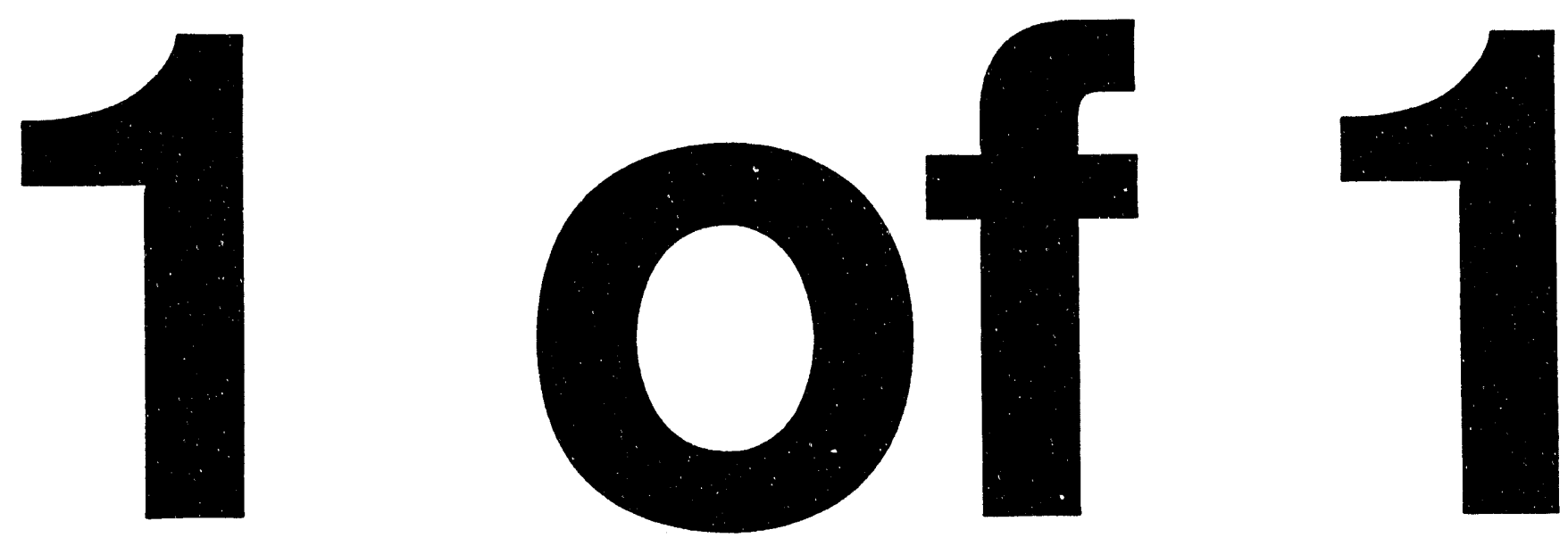


\section{La.ur. $94-1604$}

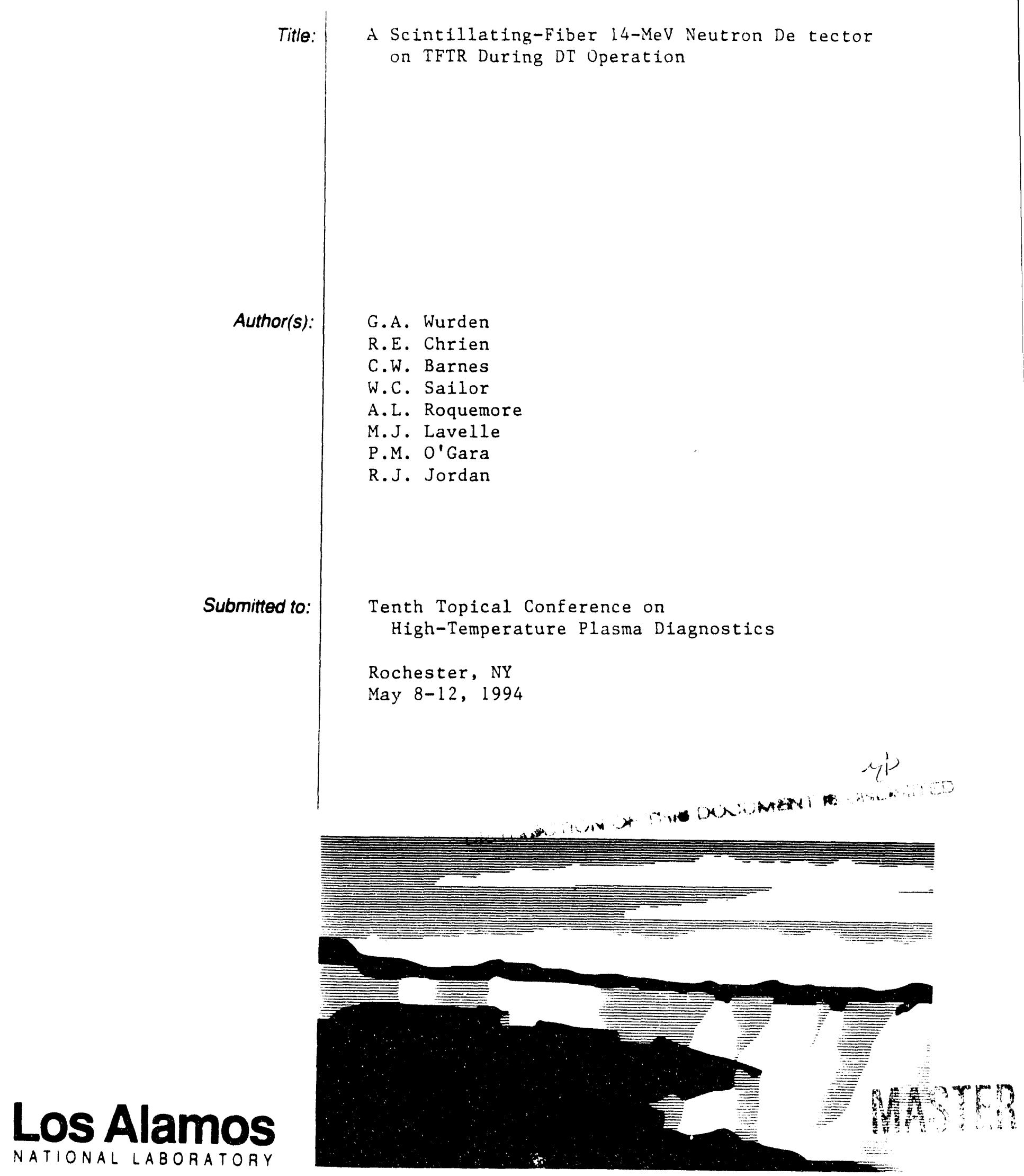

Los Alamos National Laboratory, an aftirmative actionvequal odpontunity emoidyer, is operated by the University of California tor ine U.S. Deoartment of Energy under contract W-7405-ENG-36. By acceptance of this anicie. the pubisher recognizes that the U S. Government retains a nonexciusive. royalty-free license to publish or reproduce the pubished form of inis contnbution, or to allow oihers to do requests inat the oudisher identity this anticie as work pertormed under the auspices of the U.S. Depanment of Energy. 


\title{
A Scintillating-Fiber 14-MeV Neutron Detector on TFTR during DT Operation
}

\author{
G. A. Wurden, R. E. Chrien. Cris W. Barnes, W. C. Sailor \\ Los Alamos National Laboratory, Los Alamos, NM 87544 \\ A. L. Roquemore \\ Princeton Plasma Physics Laboratory, Princeton, NJ 08543 \\ M. J. Lavelle, P. M. O'Gara, R. J. Jordan \\ EG\&G Energy Measurements, Las Vegas, NV 89125
}

\begin{abstract}
A compact $14 \mathrm{MeV}$ neutron detector using an array of scintillating fibers has been tested on the TFTR tokamak under conditions of a high gamma background. This detector uses a fiber-matrix geometry ${ }^{\prime}$, a magnetic field-insensitive phototube with an active $\mathrm{HV}$ base and pulse-height discrimination to reject low-level pulses from $2.5 \mathrm{MeV}$ neutron and intense gammas. Laboratory calibrations have been performed at EG\&G Las Veg is using a pulsed DT neutron generator and a $30 \mathrm{kCi}{ }^{60} \mathrm{Co}$ source as background, at PPPL using DT neutron sources, and at LANL using an energetic deuterium beam and target at a tandem Van de Graaff accelerator. During the first high power DT shots on TFTR in December 1993, the detector was 15.5 meters from the torus in a large collimator. For a rate of $1 \times 10^{18} \mathrm{n} / \mathrm{sec}$ from the tokamak, it operated in an equivalent background of $1 \times 10^{10} \mathrm{gammas} / \mathrm{cm}^{2} / \mathrm{sec}(\sim 4 \mathrm{~mA}$ current drain) at a DT count rate of $200 \mathrm{kHz}$.
\end{abstract}

\section{Introduction}

In magnetic fusion, the need exists for a compact, directional $14 \mathrm{MeV}$ neutron detector which can operate in a large background of gamma-rays or DD neutrons. Such a detector can be used in triton burn-up studies in DD machines, or (with sufficient radiation-hardening) in a DT fusion device as a fusion burn profile monitor. We have modeled and developed a detector which uses scintillating fibers (plastic BCF10) to achieve a directional response to incident neutrons, by keeping the diameter of the fiber small compared to the proton recoil track length in the fiber ${ }^{2}$. Directionality to neutrons using this segmented scintillator technique was demonstrated by Chupp \& Forrest ${ }^{\prime}$ in 1966 . We also employ a non-scintillating medium- $\mathrm{Z}$ matrix to reduce unwanted gamma signals that are generated by Compton electrons in the scintillator. Most of these electrons will not deposit their full energy in the fibers, but instead deposit their energy in the matrix. One can use pulse height discrimination to detect only the large signals (5 to 8.6 $\mathrm{MeV}$ electron equivalent) from forward-scattered recoil protons generated by the $14 \mathrm{MeV}$ neutrons incident along the long axis of the fiber. 


\section{Design}

The detector sensor head is $10 \mathrm{~cm}$ in length, which is approximately one e-folding length for $14 \mathrm{MeV}$ neutrons to interact in the fiber. We have constructed sensor heads with either $1-\mathrm{mm}$ diameter, or $0.5-\mathrm{mm}$ diameter Bicron BCF-10 blue scinitillating-fibers, with a relatively fast 2.4 nsec decay time. The maximum energy proton recoils from the $0^{\circ}$ events will be $2.2 \mathrm{~mm}$ in length. By using an aluminum matrix, vith fibers separated by $2.5 \mathrm{~mm}$, we can stop the recoil protons and Compton electrons from crossing from one fiber to the next. A total of 91 fibers are placed in a hexagonal grid pattern in each head. The heads are machined in $1 \mathrm{~cm}$ high blocks, and held in place by 2 indexing rods. In addition to making drilling the small diameter (but long) holes easier, this allows blocks to be removed or changed for testing purposes. We also built a black plastic (Erdylite) matrix head, which we expect would reduce the non-elastic scattering of neutrons compared to aluminum, but at the expense of having an "external" source of recoil protons adjacent to the scintillating fibers. High $\mathrm{Z}$ matrix materials are not desirable, because they cause too much angular scatter in the incident neutron flux.

We utilize an active phototube base design, incorporating Darlington-pair bipolar transistors in the last six dynode stages to supply high current output, without voltage deviations. The production circuits are preset to have up to a $5 \mathrm{~mA}$ linear output capability in steady-state. These tubes also have provided up to 4.5 Amps of linear output for brief $(100 \mathrm{nsec})$ periods. To handle the heat dissipation in the PMT base electronics of nearly 10 watts, we found it useful to have a fan provide some forced air flow across the units, especially while they are in a collimator. Due to the current demand each PMT requires its own high voltage supply at up to -2500 volts and $10 \mathrm{~mA}$ output.

Although it would be best to put the photodetector far away from the sensor head (which must operate in close proximity to the tokamak), an optical relay system would be bulky, and a non-scintillating set of transfer fibers would still generate unwanted fluorescence and noise. We chose to directly couple the phototubes to the scintillating fibers. In a typical tokamak environment, this means the tubes may be 
subjected to 500 gauss vertical fields. The optical detector must itself not generate significant noise in a harsh radiation environment, must be linear over a wide dynamic range, and must have a good quantum efficiency and gain at least of order $10^{6}$. We selected a novel Hamamatsu open mesh, 16-dynode. 2" diameter, magnetic field insensitive phototube, type R2490-05, with a star-grid on the photocathode for increased current capability. The tube and base electronics have a measured 8.6 nanosecond integral rise time, or a $10-90 \%$ rise time of $3.2 \mathrm{nsec}$, and they will continue to function in a 1 Tesla magnetic field along the tube axis, with only modest changes in gain.

The detector package is shown in FIGURE 1. The active electronics package has been moved off-axis to reduce direct line-of-sight neutron damage, assuming that supplemental collimation will be available.

A $1 / 8$ " thick lead collar which slides over the $10 \mathrm{~cm}$ long sensor head provides the only gamma shielding. A thin aluminum cover keeps out unwanted light. To reduce complications due to off-axis magnetic fields cassing some gain changes, we use a 1" soft-iron cylinder shield, and a double wrap of mu-metal, isolated from the PMT and each other by mylar wrap.

\section{Testing}

The scintillating-fiber 14-MeV neutron detector (referred to as the Sci-Fi detector), has been tested using both a laboratory DT neutron source at Princeton ${ }^{3}$, a burst DT neutron source in Las Vegas, and a tandem Van de Graaff fast neutron source at Los Alamos. In Las Vegas, we have also simulated an intense tokamak gamma background by exposing the detector to a $30 \mathrm{kCi}{ }^{60} \mathrm{Co}$ source at a distance of 3.8 meters, while also observing DT neutrons. At a PMT gain of $1.2 \times 10^{6}$, using $1 \mathrm{~mm}$ fibers in the aluminum matrix, this produced a $1 \mathrm{~mA} \mathrm{DC}$ current into a $50 \mathrm{Ohm}$ load, but did not inhibit the $100 \mathrm{MHz}$ pulse counting electronics from seeing DT neutrons.

At a distance of $35 \mathrm{~cm}$ from the PPPL DT neutron source $\left(\sim 1 \times 10^{8}\right.$ neutrons $\left./ \mathrm{sec}\right)$, using a $1-\mathrm{mm} \mathrm{Al}$ head with shiny aluminum foil reflector at the end of the fibers away from the PMT, and the PMT set at a gain of $2 \times 10^{6}$, one sees $400 \mathrm{mV}$ output pulses. The pulses are measured at the end of 30 meters of RG-58U 
cable, terminated in $50 \mathrm{Ohms}$. At a discriminator setting of $150 \mathrm{mV}$, we count 160 events/second. The detector has an end-on cross section of $0.7 \mathrm{~cm}^{2}$, so the absolute efficiency is $\sim 2.5 \%$, for an effective efficiency area product of $0.017 \mathrm{~cm}^{2}$ for DT neutrons. We can estimate the gain and efficiency of the overall system from first principles. According to Bicron, the BCF-10 fiber has a scintillation efficiency of $2.8 \%$ for minimum ionizing particles, making $3 \mathrm{eV}$ photons on average. However, only $3.4 \%$ of the light produced in the fiber is actually trapped by total internal reflection in the fiber, in each direction. So from an initial $8.6 \mathrm{MeV}_{\infty e}$ light pulse, 80,000 photons are generated, but only 2700 are trapped in the fiber. Then $480\left(18 \%\right.$ Q. E.) are detected at the PMT, and amplified as a charge of $9.6 \times 10^{8}$ electrons. This must be multiplied by 1.4 to account foil reflectivity. From the measured pulse shape, we would get a peak current of $27 \mathrm{~mA}$ into $50 \mathrm{Ohms}$, or a voltage of $1.3 \mathrm{~V}$, but then cable attenuation of $\sim 40 \%$ must be taken into account. The resulting voltage of $\sim 800 \mathrm{mV}$ is about a factor of two larger than what is actually observed. Of course there will be few pulses at the maximum energy, so a pulse height spectrum needs to be measured.

FIGURE 2 shows an angle scan of the Sci-Fi relative to the PPPL. $14 \mathrm{MeV}$ laboratory neutron source, measured on a Canberra 90 pulse height analyzer. The detector with $1 \mathrm{~mm}$ fibers shows a 5:1 pointing factor (Ratio of counts at $0^{\circ}$ to counts at $90^{\circ}$ at optimal discriminator setting), and a 30 degree HWHM angle. The "knee" feature from channels 40 to 90 (corresponding to 130 and $300 \mathrm{mV}$ respectively) is the most interesting part of the spectra, and it changes with angle. Yet higher pulse events are not angle sensitive, and we believe are caused by "end" effect events nearest the PMT, where light is more efficiently coupled to the PMT. Since the inherent fiber trapping efficiency is low, this is a serious, but not fatal defect of our present design, which therefore also admits some unwanted gamma contamination.

We also used a time of flight data acquisition system at the LANL tandem Van de Graaff ${ }^{4}$ (11.2 MeV D beam, $120 \mathrm{nA}, 1 \mathrm{nsec}$ duration, at approximately $300 \mathrm{kHz}$ rep rate, incident on a 2.5 atmosphere deuterium gas target. $1.9 \mathrm{~cm}$ in length) to see gamma and neutron responses separately. We acquired pulse height spectra for gammas from the $14 \mathrm{MeV}$ neutrons interacting in the accelerator target for the 14 
$\mathrm{MeV}$ neutrons themselves, and then for lower energy "breakup neutrons", each separately resolved by time of flight over a 3 meter flight path. The gammas extend to an energy of approximately $7.5 \mathrm{MeV}$, and are mostly from inelastic scatter in the stainless steel and gold target chamber. The effectiveness of using a segmented matrix of fibers and aluminum is seen dramatically in comparing FIGURE 3(a) to 3(b). The gamma spectrum is attenuated both in amplitude and "hardness", when comparing with/without the aluminum matrix. This is an improvement in the gamma rejection by 3 to 4 orders of magnitude over a "bulk scintillator".

\section{Prototype on TFTR}

A prototype detector was installed at TFTR, in a large existing collimator, 15.5 meters away from the plasma centerline. This detector looks through 2 " of lead, and the Surface Barrier Diode system ${ }^{5}$, in addition to structural materials of the vacuum vessel ${ }^{6}$. Nevertheless, it has provided a useful, if harsh, test of the concept. FIGURE 4 shows the response of four discriminator channeis $(50 \mathrm{mV}, 80 \mathrm{mV}, 100 \mathrm{mV}$, and $230 \mathrm{mV}$ ) from the 1 -mm fiber, black plastic "Erdylite" head. with the photomultiplier gain set at $2.5 \times 10^{6}$, driving a $75 \mathrm{ohm}$ cable with $50 \%$ pulse height attenuation. The Deuterium neutral beams were on from 3 to 4 seconds, and a tritium gas puff was introduced into the deuterium plasma at 3.5 seconds. One can see the similarity of the lowest discriminator setting to the ${ }^{235} \mathrm{U}$ fission chamber, which sees both DD and DT neutrons, and the similarity of the highest level discriminator to the Surface Barrier Diode response, which sees only the $14-\mathrm{MeV}$ DT neutrons. For discharges with greater than $10^{18}$ neutrons/sec, this head was too sensitive to gammas, and the active base would cut-off momentarily, as the current demand exceeded $4 \mathrm{~mA}$. We have since this time gone back to the aluminum matrix, with half as many 1 $\mathrm{mm}$ fibers, in order to stay on-scale for the highest power discharges.

\section{Outlook}

We have demonstrated that pulse height discrimination techniques combined with fiber optic scintillating fibers can yield a fast counting (100 MHz) directional neutron detector, sensitive to only $14 \mathrm{MeV}$ neutrons. By using a segmented matrix one can dramatically improve the gamma rejection, and by setting 
a high threshold, low energy neutrons (DD) can also be eliminated. Our testing has uncovered some problems with the fact that one should either collect ONLY the light which is trapped within the fibers, or else ALL of the light which is generated by the fibers, but not some of both. By eliminating the "end effects" close to the PMT, either through blackened aluminum matrix material or by employing a short section of "non-scintillating" fiber-faceplate material, we should be able to eliminate undesired "large pulses", and make a cleaner pulse height response. Variants of the concept could in principle be extended to radiation hardened devices suitable for compact, spatially-resolved 14-MeV neutron measurements on ITER.

We would like to acknowledge the technical efforts of Richard Scarberry and Hank Alvestead (P-1), calibration help at the Van de Graaff by George Morgan (P-15), and that this work is supported by DOE contracts W-7405-ENG-36 and DE-AC02-76-CH0-3073. 


\section{CAPTIONS}

FIGURE \#1: Sketch of the Sci-Fi detector packaging. A soft iron collar covers the phototube, and the electronics are removed to the side of the neutron flight path, in order to reduce damage to the base electronics.

FIGURE \#2: Angle scan of bare Sci-Fi detector, relative to $14 \mathrm{MeV}$ neutron source. The sensor head has $1 \mathrm{~mm}$ fibers in an aluminum matrix. The large pulses at relatively low count rates extend to channel 500 in the pulse height analyzer, and are due to undesired "end" effects, from events which send light to the PMT without having to be trapped in the optical fiber. The "knee" is the dominant feature which changes with angle of incidence.

FIGURE \#3: Comparison of the pulse height response of a 1-mm head Sci-Fi sensor to $14 \mathrm{MeV}$ neutrons and to hard gammas, without (a) and with (b) the Aluminum matrix. Lower energy neutrons, including 2.5 MeV's, are shown for reference. The gamma response overlays the $7.5 \mathrm{MeV}$ neutrons in case (a), but is near the $5 \mathrm{MeV}$ neutrons in case (b).

FIGURE \#4: Tritium Gas puff in a deuterium TFTR discharge with $15 \mathrm{MW}$ of deuterium neutral beams, showing how the response of the Sci-Fi changes as the discriminator threshold is increased. Beams are on from 3 to 4 seconds, and the gas puff occurs at 3.5 seconds. Signals from a Uranium-235 fission chamber (sensitive to both DD and DT), and a Surface Barrier Diode, sensitive to only DT neutrons are shown for comparison. 


\section{REFERENCES}

' E. I. Chupp and D. L. Forrest, IEEE Trans. Nucl. Sci., 468 (1966).

2 Accompanying paper 7.10 in this conference, W. C. Sailor, et. al.

${ }^{3}$ A. L. Roquemore, D.W. Jassby, L. Johnson, J. D. Strachan, and Cris W. Barnes, "Performance of a 14$\mathrm{MeV}$ Neutron Generator as an In-Situ Calibration Source for TFTR", Proceedings

of the IEEE/NTSS 15th Symposium on Fusion Energy, Hyannis, Oct. 1993.

${ }^{4}$ R. Woods, J. L. McKibben, and R. L. Henkel, Nucl. Inst. \& Methods, 122, 81 (1974).

${ }^{5}$ E. Ruskov, W. W. Heidebrink, H. H. Duong. A. L. Roquemore, and J. D. Strachan, paper 7.14 at this conference.

${ }^{6}$ Cris W. Barnes and J. D. Strachan, Rev. Sci. Inst.. 61, 2383, (1990). 


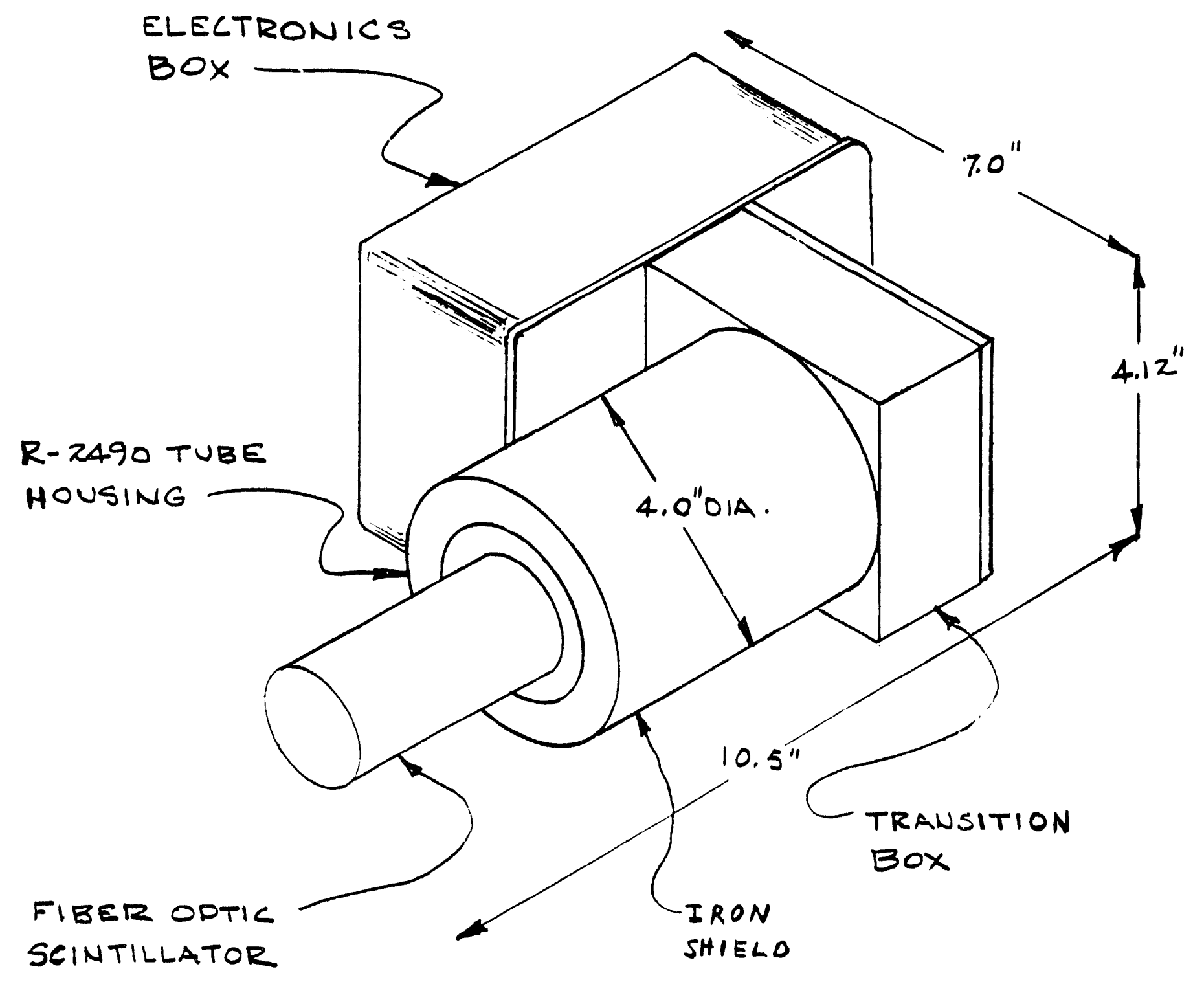
HOUSING 
ןouueyว $\forall$ Hd

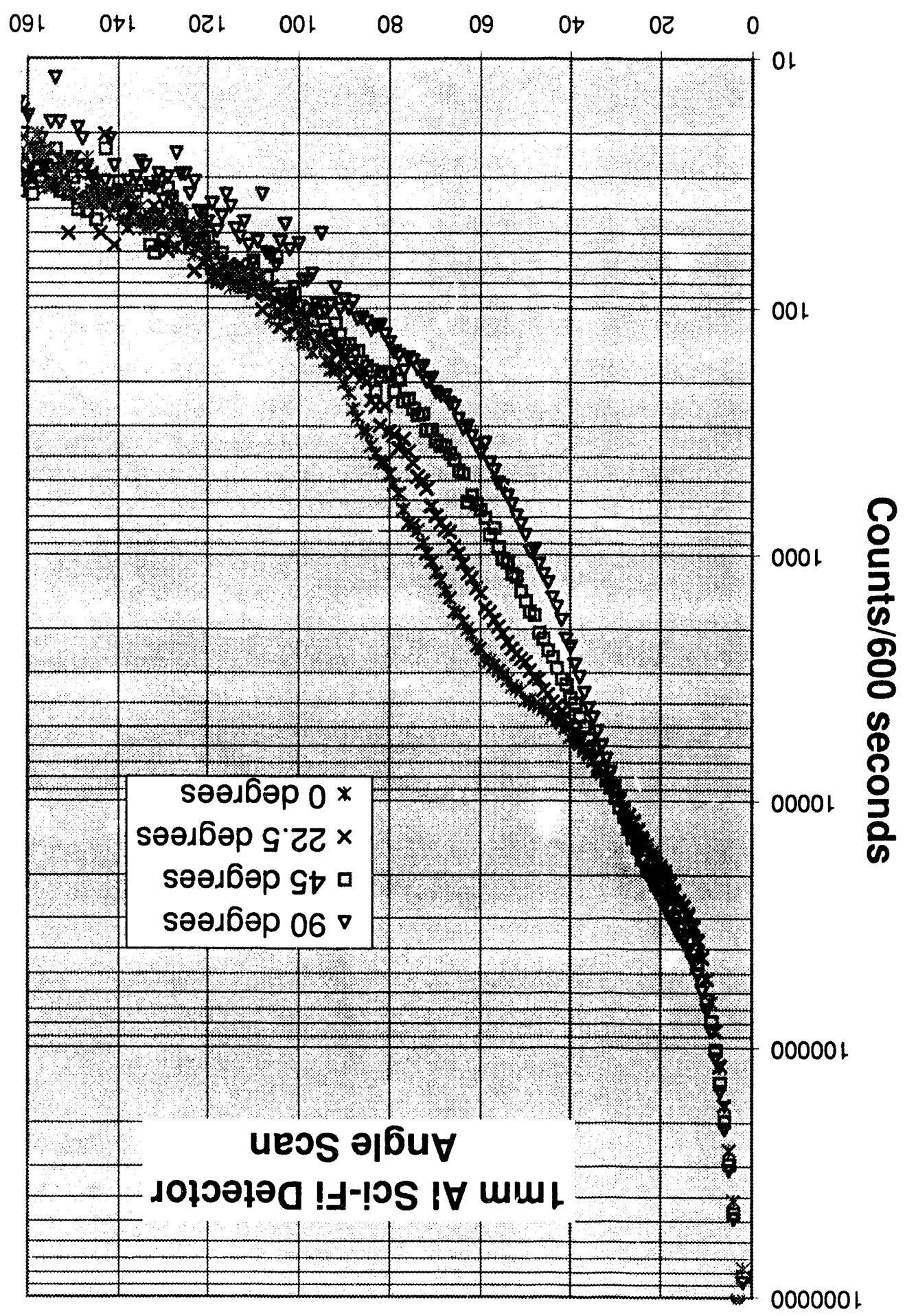




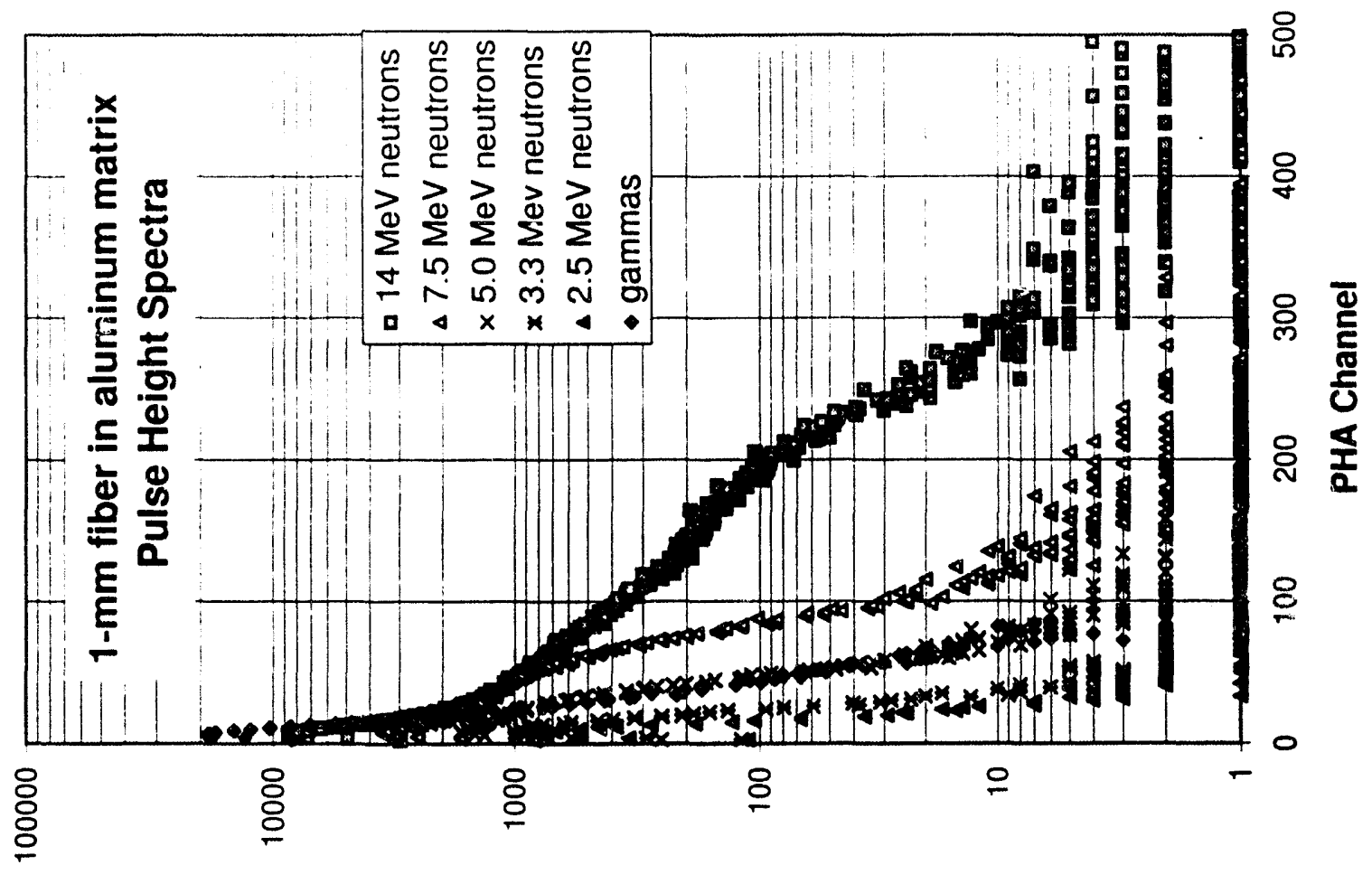

sesjnd weəg 8v0lXs/słunos
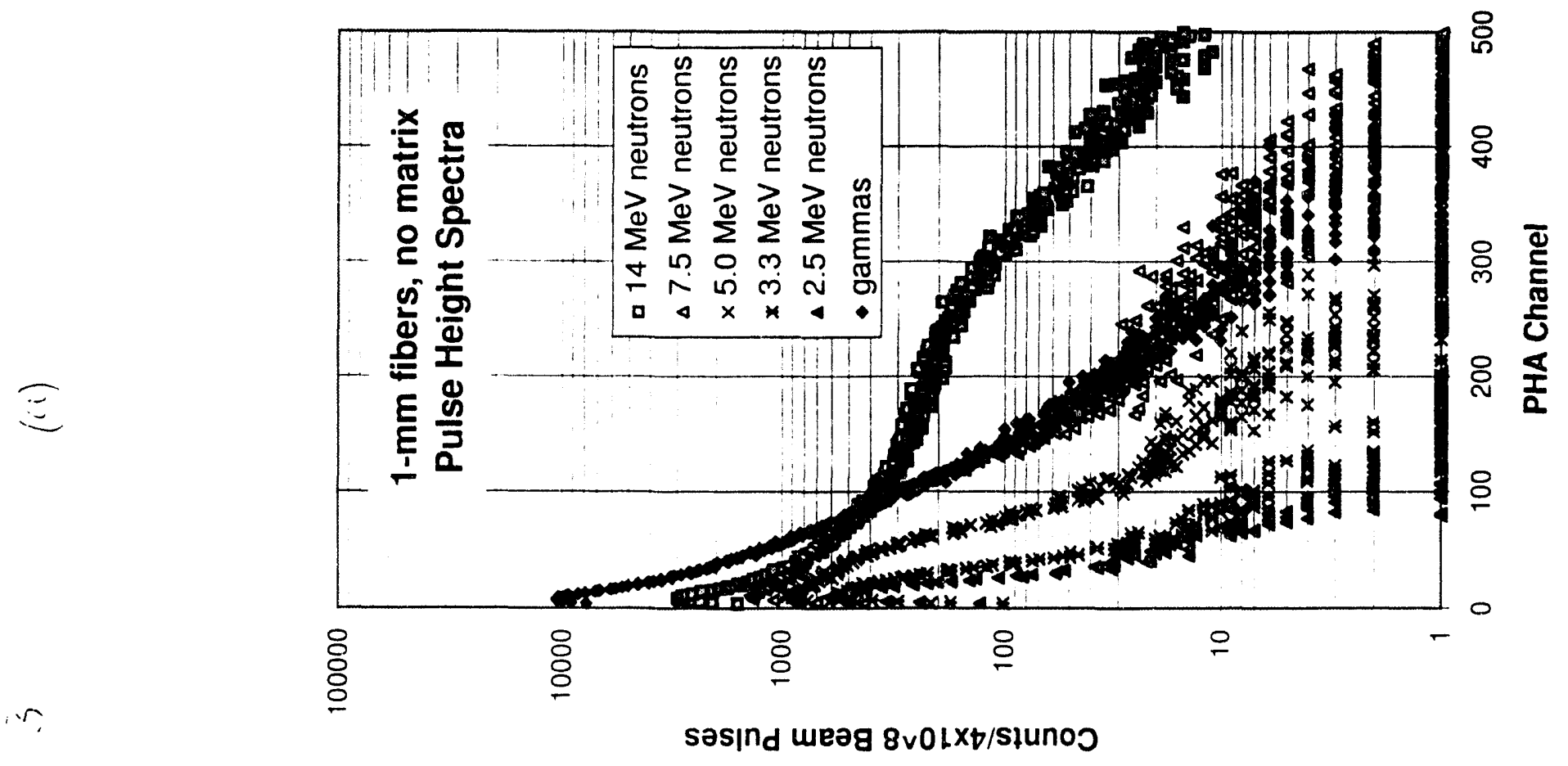

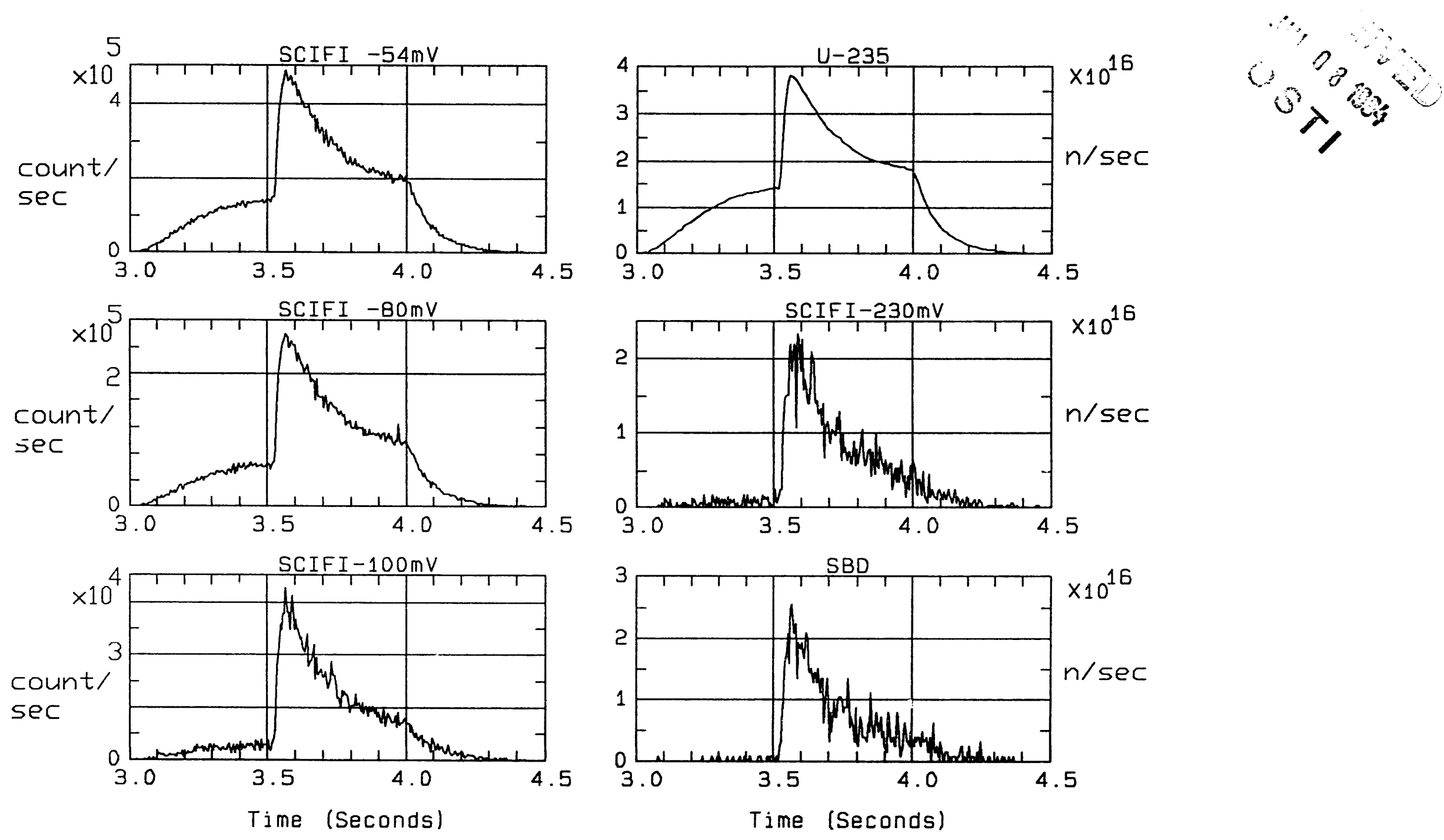

73183 12/08/93 18: 02 
DOC.I
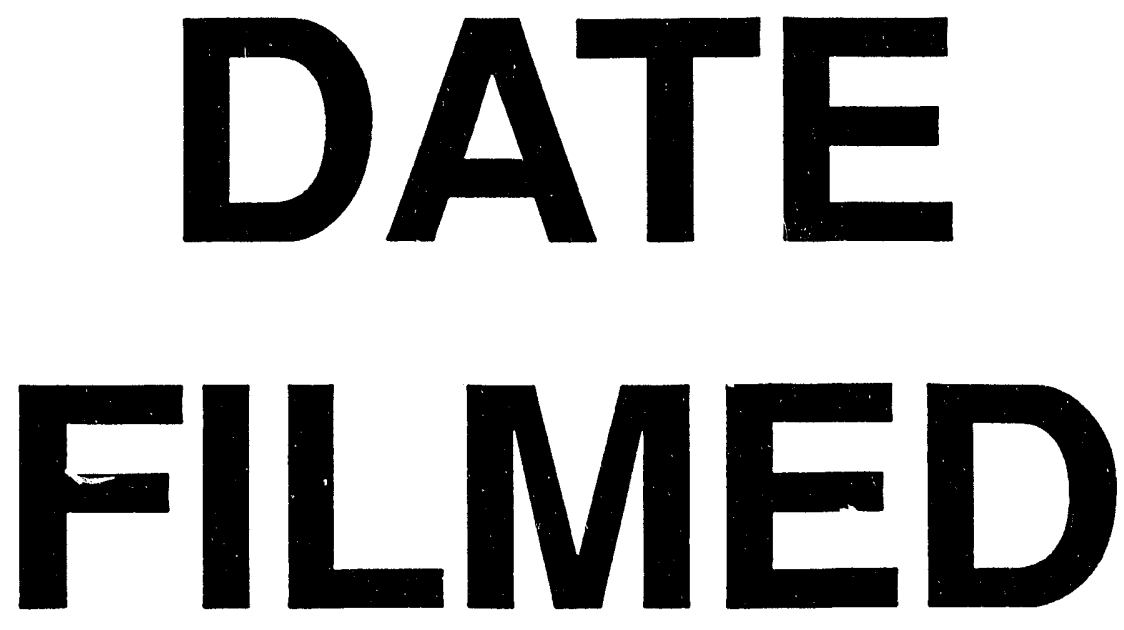

$8 / 1 / / 94$
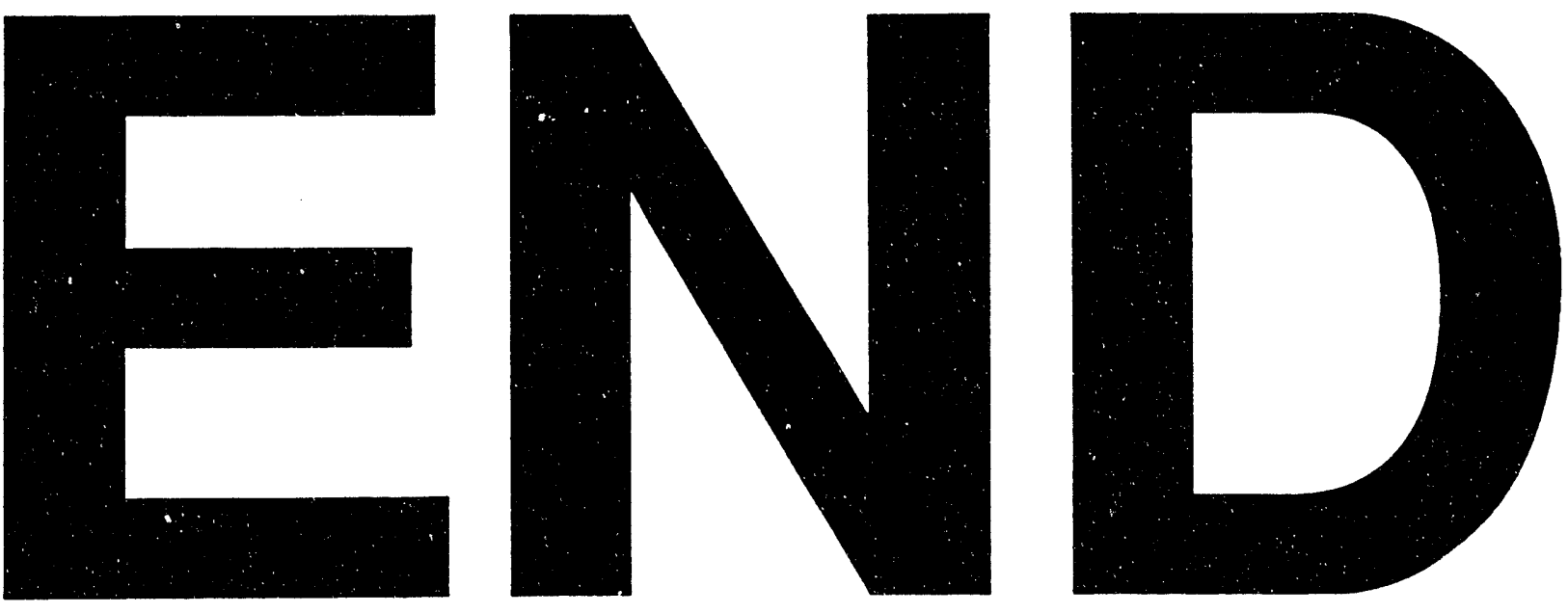
